\title{
Brief Report: Additive and Subtractive Counterfactual Reasoning of Children with High-Functioning Autism Spectrum Disorders
}

\author{
Sander Begeer • Mark Meerum Terwogt • \\ Patty Lunenburg · Hedy Stegge
}

Published online: 4 June 2009

(c) The Author(s) 2009. This article is published with open access at Springerlink.com

\begin{abstract}
The development of additive ('If only I had done...') and subtractive ('If only I had not done...') counterfactual reasoning was examined in children with High Functioning Autism Spectrum Disorders (HFASD) ( $n=72$ ) and typically developing controls $(n=71)$, aged 6-12 years. Children were presented four stories where they could generate counterfactuals based on a given consequent (e.g., 'you left muddy footprints in the kitchen. How could that have been prevented?'). Children with HFASD increasingly used subtractive counterfactuals as they got older, but controls showed an increase in additive counterfactuals, which may be linked to their growing adaptive and flexible skills. Children with HFASD likely develop different strategies for their counterfactual reasoning. The role of IQ and ideational fluency will be discussed.
\end{abstract}

Keywords Autism - Counterfactuals · Imagination · Flexibility · Fluency

\section{Introduction}

People often think about what might have been if some past event had turned out differently. This type of thinking about alternatives to past events is called counterfactual reasoning. Despite our awareness that such alternative

S. Begeer $(\bowtie) \cdot$ M. M. Terwogt $\cdot$ H. Stegge

Department of Developmental Psychology, VU University

Amsterdam, Van der Boechorststraat 1, 1081 BT Amsterdam,

The Netherlands

e-mail: S.Begeer@psy.vu.nl

P. Lunenburg

PI Research, Duivendrecht, The Netherlands events did in fact not take place, reasoning about them is an important, pervasive aspect of our daily functioning (Epstude and Roese 2008). It represents one of the defining hallmarks of the development of complex reasoning skills (Byrne 2007). In the present study we investigate the processes that underlie counterfactual reasoning, in particular the role of the imagination, of children with a disorder known to impair imaginative, social and affective functioning: autism.

Counterfactual reasoning is beneficial. It enables us to learn from own (or others) previous mistakes-preparing us to prevent bad outcomes by creating multiple options for how to handle similar situations in the future. This type of reasoning contributes to a range of cognitive processes like creativity, probability judgments, problem solving, decision-making and social functioning and has shown positive effects on task performance, satisfaction and self-esteem and alleviates depression and post-traumatic stress disorders (Epstude and Roese 2008).

The functional benefits of counterfactuals can be explained by the distinction between two ways of counterfactual reasoning. One may either subtract elements or actions from reality ('If only I had not done...') or add new elements to reality ('If only I had done...'). Subtractive counterfactual reasoning is related to analytical and problem-solving abilities, and narrowly confined to undoing elements from the original, factual set of antecedents. The search space and the number of possible subtractive counterfactuals are therefore rather limited. The structure of a subtractive counterfactual corresponds to a modus tollens inference, where the denial of an outcome (or consequence) of an inference logically results in the denial of the antecedent (Guajardo and Turley-Ames 2004). Children with ASD, in particular those with normal IQs (high functioning ASD: HFASD), are known for their 
unimpaired analytical and logical abilities, in particular regarding conditional reasoning about explicit information. They seem to resort to these strategies to compensate for the absence of more intuitive and heuristic response strategies seen in typical development (Begeer et al. in press; Peterson and Galitsky 2004; Pijnacker et al. 2009). Even mentally retarded children with ASD are skilled at conditional reasoning from explicit counterfactual antecedents (e.g., 'If A, then B. If not A, then...?') (Leevers and Harris 2000; Scott et al. 1999; Peterson and Bowler 2000). Therefore, children with HFASD are likely unimpaired in their ability to use subtractive counterfactuals.

Additive counterfactuals, on the other hand, appeal to the imagination and increase creativity and flexibility with regard to future situations. With their focus on doing something that was in fact not done, they serve a stronger preparative function than subtractive counterfactuals because they are directly related to the generation of new adaptive response options that were perhaps not considered in the past (Epstude and Roese 2008). In principle, additive counterfactuals allow an open search space and an unlimited number of responses. Impairments on this domain would fit with the defining and well documented impairments in imagination, behavioral flexibility, and spontaneous generation of new ideas (Bigham 2008; Bishop and Norbury 2005; Turner 1999). Indeed, open-ended counterfactual consequent questions showed less correct responses in 9-13 year old children with ASD and mental retardation than in controls (Grant et al. 2004). In sum, it is likely that the generation of additive counterfactuals is impaired in children with HFASD.

In the present study we focus on the development of spontaneous additive and subtractive counterfactual reasoning in children with HFASD and typically developing controls, divided over three age cohorts of $6-8,8-10$ and $10-12$ year olds. The increase in memory likely results in an age effect on the number of additive counterfactuals (Crowe and Prescott 2003). However, children with HFASD are expected to lag behind controls in their development of additive counterfactuals. Since additive counterfactual reasoning partly depends on the ability to generate new ideas, we included an ideational fluency task to investigate whether or not generative ability is a decisive factor in the use of additive counterfactuals (Bleichrodt 1988).

\section{Method}

Participants

The participants were 72 children with HFASD ( 65 boys, 7 girls) and 71 typically developing children (67 boys, 4 girls). Active parental consent was obtained for all of the participating children. The diagnostic classification of the children with HFASD was based on a diagnostic assessment by a child psychiatrist according to the DSM-IV criteria (APA 2000). None of the HFASD children had codiagnoses of additional disabilities (e.g., mental retardation, deafness) and they all came from families whose sole, or first, language was Dutch. Groups were similar in racial and ethnic breakdown and were primarily from middleclass and upper-middle-class families.

Because this research involved a language-based task, groups were matched as closely as possible on the basis of the Verbal IQ and Full-scale IQ as well as gender and chronological age. Intelligence measures were obtained in a separate session, through administration of the Dutch version of the Wechsler Intelligence Scale for Children-III (WISC-III; Kort et al. 2002). There were no significant group differences in chronological age, Verbal IQ, Full-scale IQ or ratio boys to girls (See Table 1).

\section{Procedure and Materials}

During a 30-min individual session children were administered the counterfactual antecedent task and the RAKIT idea-production task (Bleichrodt 1988). The counterfactual antecedent task consistently preceded the idea-production task. All sessions were audio taped and later transcribed. The transcriptions were scored by two independent coders.

\section{Counterfactual Antecedent Task}

The counterfactual antecedent task (Guajardo and TurleyAmes 2004) consisted of four stories, for example: 'Imagine that you are playing outside in the muddy yard. You are thirsty so you go inside to the kitchen to get a drink of juice. You walk through the mud, you step over the doormat, and you keep your shoes on. Because your shoes are muddy, you get dirt all over the floor.' Then the children were asked, 'What could you have done so that the kitchen floor would not have gotten dirty?'. After each generated antecedent children were asked, 'Can you think of anything else?'. Children were encouraged to generate as many counterfactual antecedents as possible.

RAKIT idea-production task (Bleichrodt 1988) was included in this study as a measure of ideational fluency. This task consisted of five different questions (e.g., 'What can you see in a shop?' or 'What can you drink?'). Children were asked to generate as many ideas within a certain category as possible. 
Table 1 Details of the participants (means, SDs and ranges)
HFASD high functioning autism spectrum disorders; $C A$ chronological age; VIQ verbal IQ; $P I Q$ performal IQ; FSIQ full scale IQ; $S D$ standard deviation

\begin{tabular}{lllll}
\hline & CA (years;months) & VIQ & PIQ & FSIQ \\
\hline 6-8 Years & & & & \\
HFASD $(N=22)$ & $7 ; 2(0 ; 6)$ & $97.3(8.9)$ & $82.3(13.2)$ & $97.4(12.4)$ \\
& $6 ; 1-8 ; 0$ & $82-109$ & $76-101$ & $75-117$ \\
Comparison $(N=24)$ & $7 ; 5(0 ; 5)$ & $100.0(13.0)$ & $100.1(13.5)$ & $100.0(13.4)$ \\
& $6 ; 5-8 ; 1$ & $80-127$ & $79-130$ & $79-128$ \\
8-10 Years & & & & \\
HFASD $(N=21)$ & $8 ; 9(0 ; 5)$ & $108.7(15.6)$ & $106.7(14.1)$ & $107.0(14.2)$ \\
& $8 ; 1-9 ; 9$ & $77-144$ & $78-133$ & $84-145$ \\
Comparison $(N=21)$ & $8 ; 9(0 ; 5)$ & $102.7(11.6)$ & $102.3(11.6)$ & $102.4(11.7)$ \\
& $8 ; 2-9 ; 9$ & $79-121$ & $77-123$ & $78-121$ \\
10-12 Years & & & & $104.0(15.9)$ \\
HFASD $(N=29)$ & $11 ; 0(0 ; 8)$ & $104.1(19.1)$ & $100.8(15.9)$ & $78-138$ \\
& $10 ; 1-12 ; 8$ & $77-150$ & $72-132$ & $108.9(16.9)$ \\
Comparison $(N=26)$ & $11 ; 2(0 ; 7)$ & $108.9(16.7)$ & $108.8(17.0)$ & $70-139$ \\
\hline
\end{tabular}

\section{Scoring}

\section{Counterfactual Task}

Children received 1 point for each counterfactual antecedent they generated. Subsequently, all antecedents were categorized as additive (if children added an element, e.g., 'wipe your feet') or subtractive (if they removed an element, e.g., 'not going outside to play'). Inter-rater reliability was satisfactory (Kendall's $\tau=.77$ ).

RAKIT idea-production task. Scoring methods were used as described in the RAKIT manual (Bleichrodt 1988). Children received one point for each plausible response, and scores on the five questions were summed. Inter-rater reliability was satisfactory (Kendall's $\tau=.74$ ).

\section{Results}

A 2 (Group: control, HFASD) $\times 2$ (Structure: additive, subtractive) $\times 3$ (Age: 6-8, 8-10, 10-12) MANOVA, controlling for full scale IQ was conducted to examine the effect of age and diagnosis on the number of additive and subtractive counterfactuals that were generated. There were significant effects of Age $(F(2,136)=15.88, p<.01$, $d=.66)$ and interactions for Structure $\times$ Age $(F(1$, 134) $=7.36, p<.01, d=.45)$, which were qualified by an interaction of Group $\times$ Age $\times$ Structure $(F(2,136)=4.63$, $p=.05, d=.36)$.

Post-hoc analysis, controlling for full scale IQ, showed that for children with HFASD there was an effect of Age on generating subtractive counterfactuals $(F(2,68)=3.63$, $p<.05, d=.32)$, but not on additive counterfactuals ( $F(2$, $68)=1.21$, n.s.). In the control group there was a main effect of Age on generating additive counterfactuals $(F(2$, $67)=15.25, p<.001, d=.65)$, but not on subtractive counterfactuals $(F(2,67)=.97$, n.s. $)$. Opposite group differences were found in the youngest and the oldest age samples. In the youngest age group, children with HFASD generated less subtractive counterfactuals than controls $(F(1,43)=5.12, p<.05, d=.66)$. However, when covarying for non-verbal IQ skills, which were found to differ in the youngest age cohort, this effect was reduced to a trend $(F(1,43)=2.84, p<.08, d=.50)$. Within the oldest age group children with HFASD generated less additive counterfactuals than controls $(F(1,52)=4.73$, $p<.05, d=.58)$.

A 2 (Group: control, HFASD) × 3 (Age: 6-8, 8-10, 10-12) ANOVA was conducted to examine differences between children with HFASD and controls on the number of ideas they generated on the RAKIT idea-production task. Main effects of Group $(F(1,136)=7.13, p<.01$, $d=.45)$ and Age $(F(1,136)=19.06, p<.001, d=.73)$ indicated that children with HFASD generated fewer ideas than controls and the mean number of ideas increased with age in all children (see Table 2). Ideational fluency was strongly correlated to additive counterfactuals in both groups (HFASD: $r=.43, p<.001$, controls: $r=.59$, $p<.001$ ), but no correlations were found with subtractive counterfactuals. The correlation between ideational fluency and additive counterfactuals remained significant after controlling for full scale IQ (HFASD: $r=.36, p<.001$, controls: $r=.55, p<.001)$. Moreover, after controlling for differences in ideational fluency, the effects of the counterfactual task remained significant, with a Group $\times$ Age $\times$ Structure interaction, $F(2,135)=4.87, p<.01$, $d=.53$, and age effects on additive counterfactuals in the control group, $F(1,66)=6.44, p<.01, d=.61$, and on 
Table 2 Mean and standard deviation of the total number of additive and subtractive counterfactual antecedents children generated in response to four stories (range $0-\infty$ )

\begin{tabular}{|c|c|c|c|c|c|c|}
\hline & \multicolumn{3}{|l|}{ HFASD } & \multicolumn{3}{|l|}{ Control } \\
\hline & $\begin{array}{l}6-8 \text { Years } \\
n=25\end{array}$ & $\begin{array}{l}8-10 \text { Years } \\
n=21\end{array}$ & $\begin{array}{l}10-12 \text { Years } \\
n=30\end{array}$ & $\begin{array}{l}6-8 \text { Years } \\
n=24\end{array}$ & $\begin{array}{l}8-10 \text { Years } \\
n=21\end{array}$ & $\begin{array}{l}10-12 \text { Years } \\
n=25\end{array}$ \\
\hline Additive & $6.64(3.12)$ & $8.10(3.03)$ & $8.37(2.19)$ & $5.58(1.35)$ & $9.05(3.68)$ & $10.04(2.73)$ \\
\hline Subtractive & $.60(.50)$ & $1.29(.96)$ & $1.23(.86)$ & $1.00(.72)$ & $1.14(.79)$ & $1.00(1.16)$ \\
\hline Idea production & 31.84 (13.38) & $43.24(18.15)$ & $53.17(18.40)$ & $37.16(13.38)$ & $50.33(12.68)$ & $60.23(17.70)$ \\
\hline
\end{tabular}

subtractive counterfactuals in the HFASD group $F(2$, $67)=4.47, p<.05, d=.51$.

\section{Discussion}

Overall, children with HFASD reported an equal number of counterfactuals, both additive and subtractive, compared to controls. This seems in line with earlier findings on adept counterfactual consequent reasoning in children with ASD and mental retardation (Leevers and Harris 2000; Scott et al. 1999). However, this finding was qualified by contrasting age effects in the HFASD and control samples. With age, children in the HFASD group showed a modest increase in their generation of subtractive counterfactuals, a finding that was absent in the control group. The control group, on the other hand, showed an increase in the use of additive counterfactuals. Both findings were independent of cognitive ability and ideational fluency. In addition, compared to age matched controls, the 6-8 year old HFASD group generated less subtractive counterfactuals, while the 10-12 year old HFASD group generated less additive counterfactuals.

The stable number of additive counterfactuals in HFASD children over the three age cohorts indicates different developmental trajectories with regard to generating new plans when reasoning about alternative realities. Although the ideational fluency of HSFASD groups increased linearly with age, there was no corresponding increase in the generation of additive counterfactuals. Instead, even within the limited search space of subtractive counterfactuals, HFASD children showed an increased tendency to subtract elements from the factual information as they got older. This may be related to an acquired ability to inhibit factual information, which typically develops during this period (Bartgis et al. 2008). It should be noted that the increase in subtractive counterfactuals of HFASD was modest. It did not rise above the level of the control group, and the 6-8 year old HFASD children did in fact generate less subtractive counterfactuals than controls. The onset of using subtractive counterfactuals may occur later in HFASD than in typically developing children. However, this is an assumption that needs to be tested, possibly with a bigger search space, allowing more subtractive responses. This way, it could be confirmed whether a stable use of additive counterfactuals remains in contrast with a possible increase in subtractive counterfactuals in older HFASD children. Such a finding would fit with the preference of individuals with HFASD for explicit information and logic reasoning. Also, their tendency to 'think within the box' (Begeer et al. 2007), that is, to narrow responses down to the explicitly given information fits with the use of subtractive counterfactuals, which can be deductively negated by applying a simple logical algorithm, rather than inductively generating new responses (Klin et al. 2002). Indeed, the autistic mind seems suited to work within a closed system of specified, top down rules (Baron-Cohen et al. 2003).

As expected (Turner 1999), HFASD children showed a limited capacity for general idea production, but this element per se does not seem a sufficient explanation for the use of additive counterfactuals. The latter induces the use of generative abilities within the restriction of the factual content of the story. This requires a child to navigate between factual and alternative realities. The executive ability to switch between different perspectives, alternating between inhibiting the factual or counterfactual reality may have prevented more adequate use of their imaginative skills. Future studies will have to more closely delineate the role of executive functions in counterfactual reasoning.

Furthermore, a complicating factor for the HFASD children may have been to first override the factual information and then generate new ideas. The factual information cues a strong explicit prime. While their cognitive abilities may have enabled them to deny this information, the uncued, spontaneous generation of alternative response options may have required too much of their cognitive flexibility. Indeed, fluency performance of HFASD children is most disrupt when words are uncued (Boucher 1988), and additive responses can be compared to a response to open end questions, which raise problems for individuals with ASD (Turner 1999).

Among the limitations of the current study is the absence of longitudinal data. The current cross-sectional 
design provides a strong hypothesis for a divergent development of additive and subtractive counterfactuals in children with ASD and controls, but the only way to put this hypothesis to the test is following children over time. Furthermore, though the current data are based on spontaneous reports of counterfactual thinking, the question remains whether real life situations do indeed elicit counterfactual thinking to the same extent in both groups. Future studies are needed to provide more accurate measures of real life use of counterfactual thought and its relation to adaptive reasoning and behavior.

Acknowledgments We thank the teachers and children of the participating schools for their time.

Open Access This article is distributed under the terms of the Creative Commons Attribution Noncommercial License which permits any noncommercial use, distribution, and reproduction in any medium, provided the original author(s) and source are credited.

\section{References}

American Psychiatric Association. (2000). Diagnostic and statistical manual of mental disorders (4th ed.). Washington, DC: Author.

Baron-Cohen, S., Richler, J., Bisarya, D., Gurunathan, N., \& Wheelwright, S. (2003). The systemizing quotient: An investigation of adults with Asperger syndrome or high-functioning autism, and normal sex differences. Philosophical Transactions of the Royal Society of London. Series B, Biological Sciences, 358, 361-374. doi:10.1098/rstb.2002.1206.

Bartgis, J., Thomas, D. G., Lefler, E. K., \& Hartung, C. M. (2008). The development of attention and response inhibition in early childhood. Infant and Child Development, 17, 491-502. doi:10.1002/icd.563.

Begeer, S., Meerum-Terwogt, M., Rieffe, C., Stegge, H., Olthof, T., \& Koot, H. M. (in press). Brief report: Understanding emotional transfer in children with autism spectrum disorders. Autism.

Begeer, S., Terwogt, M. M., Rieffe, C., Stegge, H., \& Koot, H. M. (2007). Do children with autism acknowledge the influence of mood on behaviour? Autism, 11, 503-521. doi:10.1177/1362 361307083262.

Bigham, S. (2008). Comprehension of pretence in children with autism. The British Journal of Developmental Psychology, 26, 265-280. doi:10.1348/026151007X235855.

Bishop, D. V. M., \& Norbury, C. F. (2005). Executive functions in children with communication impairments, in relation to autistic symptomatology-I: Generativity. Autism, 9, 7-27. doi:10.1177/ 1362361305049027.
Bleichrodt, N. (1988). RAKIT: Handleiding bij de revisie amsterdamse kinder intelligentie test. Lisse: Swets \& Zeitlinger.

Boucher, J. (1988). Word fluency in high-functioning autisticchildren. Journal of Autism and Developmental Disorders, 18, 637-645. doi:10.1007/BF02211881.

Byrne, R. M. J. (2007). Precis of the rational imagination: How people create alternatives to reality. The Behavioral and Brain Sciences, 30, 439-480.

Crowe, S. J., \& Prescott, T. J. (2003). Continuity and change in the development of category structure: Insights from the semantic fluency task. International Journal of Behavioral Development, 27, 467-479. doi:10.1080/01650250344000091.

Epstude, K., \& Roese, N. J. (2008). The functional theory of counterfactual thinking. Personality and Social Psychology Review, 12, 168-192. doi:10.1177/1088868308316091.

Grant, C. M., Riggs, K. J., \& Boucher, J. (2004). Counterfactual and mental state reasoning in children with autism. Journal of Autism and Developmental Disorders, 34, 177-188. doi:10.1023/ B:JADD.0000022608.57470.29.

Guajardo, N. R., \& Turley-Ames, K. J. (2004). Preschoolers' generation of different types of counterfactual statements and theory of mind understanding. Cognitive Development, 19, 5380. doi:10.1016/j.cogdev.2003.09.002.

Klin, A., Jones, W., Schultz, R., Volkmar, F., \& Cohen, D. (2002). Defining and quantifying the social phenotype in autism. The American Journal of Psychiatry, 159, 895-908. doi:10.1176/ appi.ajp.159.6.895.

Kort, W., Schittekatte, M., Compaan, E. L., Bosmans, M., Bleichrodt, N., Vermeir, G., et al. (2002). WISC-III NL. Handleiding. London: The Psychological Corporation.

Leevers, H. J., \& Harris, P. L. (2000). Counterfactual syllogistic reasoning in normal 4-year-olds, children with learning disabilities, and children with autism. Journal of Experimental Child Psychology, 76, 64-87. doi:10.1006/jecp.1999.2542.

Peterson, D. M., \& Bowler, D. M. (2000). Counterfactual reasoning and false belief understanding in children with autism. Autism, 4, 391-405. doi:10.1177/1362361300004004005.

Peterson, D., \& Galitsky, B. (2004). Handling default rules by autistic reasoning. Knowledge-Based Intelligent Information and Engineering Systems, Pt 3. Proceedings, 3215, 314-320.

Pijnacker, J., Hagoort, P., Buitelaar, J., Teunisse, J. P., \& Geurts, B. (2009). Pragmatic inferences in high-functioning adults with autism and asperger syndrome. Journal of Autism and Developmental Disorders, 39, 607-618.

Scott, F. J., Baron-Cohen, S., \& Leslie, A. (1999). 'If pigs could fly': A test of counterfactual reasoning and pretence in children with autism. The British Journal of Developmental Psychology, 17, 349-362. doi:10.1348/026151099165339.

Turner, M. A. (1999). Generating novel ideas: Fluency performance in high-functioning and learning disabled individuals with autism. Journal of Child Psychology and Psychiatry and Allied Disciplines, 40, 189-201. doi:10.1111/1469-7610.00432. 